\title{
O INSTRUMENTALISMO NA AFERIÇÃO DA EXPERIÊNCIA DO USUÁRIO
}

\author{
INSTRUMENTALISM IN USER EXPERIENCE ASSESSMENT
}

\author{
Marcelo Pereira Demilis', Richard Perassi Luiz de Sousa', Giselle Alves Diaz \\ Merino ${ }^{1}$
}

RESUMO: Instrumentalismo é o nome de uma doutrina no campo de Epistemologia que indica que conhecimento e método científico não são necessariamente falsos ou verdadeiros. Mas, devido ao seu caráter instrumental, esses servem de recursos para lidar com a realidade. Por sua vez, como aspecto da realidade prática na área de Design, observa-se a busca por métodos e outros recursos para mensurar a experiência do usuário (UX), porque, progressivamente, aumentou o interesse em design orientado para a experiência. Contudo, as experiências abrangem uma gama de propriedades e métricas que são variadas, muitas vezes particulares, efêmeras e subjetivas. Por isso, há dificuldades para se encontrar conceitos que designam a experiência do usuário e, consequentemente, recursos instrumentais relacionados com essa temática. Isso caracteriza a problemática desta pesquisa sobre o instrumentalismo na aferição da experiência do usuário. O objetivo foi identificar instrumentos que, de maneira eficiente, auxiliam os designers na projeção e mensuração da experiência do usuário, visando o desenvolvimento coerente de produtos e serviços. O procedimento adotado inicialmente no estudo proposto, como é descrito neste artigo, foi pesquisar trabalhos publicados em periódicos internacionais por meio de uma revisão sistemática. O conhecimento resultante permitiu a sistematização da literatura e a seleção de instrumentos considerados coerentes com o propósito da pesquisa. Portanto, o resultado foi o mapeamento descritivo de instrumentos destinados à aferição da experiência do usuário, para serem aplicados em projetos de design.

PALAVRAS-CHAVE: Experiência do Usuário; Instrumentos de Pesquisa; Revisão Sistemática; Circunstâncias de Uso; Projeto de Design.

ABSTRACT: Instrumentalism is the name of a doctrine in the field of Epistemology, which indicates certain knowledge and scientific methods are not necessarily false or true. But due to their instrumental character, they serve as tools to handle reality. In turn, as an aspect of practical reality in the Design field, one observes the search for methods and other resources to measure user experience (UX), since, progressively, there was an increase in interest in experience-oriented Design. However, experiences have various characteristics and metrics, often singular, ephemeral and subjective. Hence, it is difficult to find concepts that project user experience and, consequently, instrumental resources related to this theme. This characterizes the problematics of this research on instrumentalism in the measurement of user experience. The objective was to identify instruments that, in an efficient way, help designers in the projection and measurement of the user experience, aiming for the coherent development of products and services. The procedure adopted initially in the proposed study, as described in this article, was to research in articles published in international journals through a systematic review. The resulting knowledge allowed for a systematization of the literature and a selection of instruments considered coherent with the purpose of the research. The result obtained from the research was the descriptive mapping of instruments for determining user experience, to be applied in Design projects.

KEYWORDS: User Experience; Research Instruments; Systematic review; Circumstances of Use; Design Project.

Fonte de financiamento: Capes

Conflito de interesse: Declararam não haver

Submetido em: 28/12/2017 Aceito em: 18/03/2018 


\section{INTRODUÇÃO}

O instrumentalismo de Dewey traz a concepção de que ciência e conhecimento em geral devem ser pensados para atender às necessidades humanas (SANTOS, 2012). O mesmo princípio rege as ciências socialmente aplicadas e a tecnologia, que é seu produto. No campo tecnológico de Design, os designers devem se preocupar com as necessidades sociais e, atualmente e cada vez mais, também conhecer as demandas do usuário para a concepção de seus projetos (BONSIEPE, 2012; MUNARI, 2008). No desenvolvimento da área de Design, durante várias décadas, buscou-se predominantemente antecipar as necessidades prático-funcionais do usuário com ideias autorais e inovadoras (LÖBACH, 2001). Assim, eram previamente e idealmente propostos projetos e produtos que determinariam novas utilizações, alterando o cotidiano de usuários e consumidores. Isso ainda acontece com frequência, especialmente diante da constante demanda por inovação. Porém, em decorrência da ampla oferta de produtos e serviços, como soluções eficientes para situações prático-funcionais, esse público passou a demandar e ser atendido com benefícios sensoriais, emocionais e simbólicos, no processo experiencial de utilização e consumo de bens e serviços (HASSENZAHL, 2010; IIDA; BUARQUE, 2016; LÖBACH, 2001).

Há, portanto, uma realidade mais recente, na qual os usuários requerem benefícios para além da funcionalidade, inclusive, evocando o direito à felicidade. Assim, entre outros profissionais, os designers têm investido no atendimento de necessidades simbólico-afetivas dos usuários, propondo-lhes experiências de felicidade por meio de produtos e serviços, nos campos material e digital (OLIVEIRA; LIMEIRA; SANTA-ROSA, 2014). Desse modo, o conhecimento sobre as experiências dos usuários, sejam essas atuais, simuladas ou projetadas, passou a ser prioridade e, atualmente, os designers projetam enfatizando a experimentação, inclusive, utilizando recursos baseados em tentativa e erro, intuições e acasos (OLIVEIRA; LIMEIRA; SANTA-ROSA, 2014; PANTALEÃO; PINHEIRO, 2009).

Segundo Hassenzahl (2004) o conhecimento das demandas do usuário está ainda relacionado a suas necessidades hedônicas no contexto de suas experiências. Por tratar o pensamento como um modo de agir sobre as coisas, o instrumentalismo funciona como um mecanismo constituidor de nossas experiências. Assim, é conceituado por Japiassú e Marcondes (1996, p. 71) como "ato de recorrer à experiência concreta (de ordem perceptual, intuitiva, ativística, axiológica ou mística) na fonte da verdade”. Isso também pode ser expandido para considerar a experiência do usuário (UX) como uma resultante da interação homem-produto (HODZA, 2009), sendo que o produto pode ser um objeto, ou um conjunto de objetos e performances caracterizando um serviço, uma interface ou um ambiente, tornando possível a conexão com o pensamento de Dewey (1978), propondo que experiência resulta da interação indivíduo-ambiente.

Os produtos de design são desenvolvidos com base nos conceitos e arranjos científicos promovendo experiências sensoriais que, também, podem mudar as ideias humanas (CAMPOS et al., 2015). No seu cotidiano, as pessoas em geral utilizam instrumentos conceituais e materiais para realizar ações e atingir objetivos (CUPANI, 2004). Por sua vez, os designers também necessitam de instrumentos conceituais e materiais para projetarem os recursos que promovem uma experiência. Contudo, há também ampla variedade e grande quantidade de produtos disponíveis, sendo que isso impõe a constante necessidade de seu aprimoramento por meio da adaptação cada vez melhor de produtos e serviços à experiência do usuário. Portanto, há o interesse de, cada vez mais, aprimorar as experiências, porque um produto ou serviço melhor adaptado ao usuário também é algo inovador, tendendo a ser percebido com maior valor.

Em síntese, na área de Design, desde o início o interesse de seus profissionais foi voltado à experiência do usuário. Contudo, anteriormente, a prioridade era antecipar idealmente das necessidades e soluções para promover experiências prático-funcionais positivas (LÖBACH, 2001). Atualmente, o interesse é primeiramente conhecer a realidade e a potencialidade experiencial do usuário, especialmente para investir na inovação ou no aprimoramento simbólico-afetivo 
das experiências. É por isso que se afirma o recente crescimento dos projetos de design orientados à experiência (TONETTO; DESMET, 2016).

No geral, o conceito de experiência é relacionado aos cinco sentidos: tato, olfato, visão, paladar e audição. Por sua vez, os sentidos reagem a alguma forma de estímulo (FERREIRA, 2011). Dewey (1959) propõe que o conceito de experiência vai além, colaborando na instauração ou na manutenção de hábitos. Assinala também a continuidade da experiência, que não tem começo nem fim, apresentando-se como um todo, um fluxo apreendido por meio de nossos sentidos em um movimento de estabelecer e expandir certos padrões nas ações. De maneira mais específica, a experiência do usuário é projetável, sendo construída de elementos ou aspectos subjetivos e efêmeros (HASSENZAHL; DIEFENBACH; GÖRITZ, 2010; LAW et al., 2009). Por isso, cabe ao designer se munir de ferramentas que o possibilitem entender e tentar prever a experiência do usuário, visando o desenvolvimento de produtos, interfaces digitais ou serviços que atendam às necessidades do usuário e sejam positivamente significativas. Segundo Lorieri (2000), o conteúdo significativo de uma experiência é medido pelas relações ou conexões nela presentes e que precisam ser captadas pela inteligência, pela "leitura interna" feita por esta função natural.

A inteligência humana pode ser considerada um instrumento, que, na filosofia instrumental, resolve os problemas à medida que surgem (DEWEY, 2008). Um instrumento é um recurso para atingir um resultado. Pode ser um objeto simples ou constituído de várias partes que serve para executar determinada tarefa, medição ou observação. Sob o viés da Engenharia, a área de Design assume seu caráter prático, cujos aspectos técnicos são ensinados e desenvolvidos de modo instrumental, como ferramentas. O objetivo é materializar as ideias de maneira eficaz e transdisciplinar para satisfazer as necessidades latentes de um público (AZEVEDO, 2006; BOMFIM, 1997; DWEK; COUTINHO; MATHEUS, 2011; KOTLER; KELLER, 2006). No recorte deste estudo, o conceito de "instrumento" envolve tudo que é limitado e serve para executar algum trabalho ou tarefa, seja física ou mental, podendo ser observação, interpretação ou ação física, incluindo um conjunto ou um procedimento específico, visando alcançar algum resultado (BUNGE, 1969, 1985; CUPANI, 2004). De maneira geral, métodos e modelos e outros instrumentos complexos envolvem um sistema de recursos conceituais, procedimentos ou ações, bem como artefatos.

Como profissionais do campo tecnológico, os designers se interessam por modelos constituídos por recursos instrumentais, que devem permitir o conhecimento e o controle sobre a experiência do usuário, especialmente quando se trata de reconhecer e propor experiências afetivamente memoráveis e satisfatórias. Assim, os designers afastam-se dos procedimentos exclusivamente intuitivos para desenvolver ou conhecer recursos instrumentais que, de maneira lógica, sistemática, sirvam para conhecer ou promover experiências interessantes aos usuários. Dessa forma, primeiramente, os designers pesquisadores adotaram conceitos e recursos instrumentais de Ciências Sociais para realizarem a mensuração da experiência do usuário, antes de haver adaptações e desenvolvimento de um repertório característico da área de Design (LAURANS et al., 2009; POELS; DEWITTE, 2006; TONETTO; DESMET, 2016). A diversidade e abrangência das experiências envolvem uma ampla quantidade de propriedades e métricas muito variadas, sendo muitas vezes particulares, efêmeras e subjetivas (HASSENZAHL; DIEFENBACH; GÖRITZ, 2010; LAW et al., 2009), porque são condicionadas por fatores humanos (GREEN; JORDAN, 1999). Isso impõe dificuldades à proposição dos conceitos e instrumentos que delimitem e identifiquem aspectos componentes da ampla e diversificada experiência do usuário.

Por essa razão, tendo como alicerce a perspectiva instrumentalista que sustenta que o desenvolvimento científico (teórico) e tecnológico (prático) é a ferramenta que o homem controla para satisfazer suas necessidades; e ponderando que Feenberg (2010), com base em DEWEY (1959), propõe que os instrumentos sejam avaliados em dois níveis considerando que a tecnologia é estudo sistemático (científico) de técnicas, processos, métodos, meios e instrumentos: 
1) considera-se a funcionalidade do instrumento que, quando aplicado, deve cumprir com eficiência a tarefa prevista. Isso requer a retirada do instrumento de seu contexto social, avaliando especificamente suas propriedades utilitárias;

2) são considerados os aspectos de design e implementação da tecnologia em interação com sistemas e dispositivos previamente existentes e de acordo com as condições naturais e sociais de seu contexto.

Deste modo, desenvolveu-se esta pesquisa. Objetivou-se, por meio de um recorte de uma revisão sistemática sobre experiência do usuário e fatores humanos/ ergonomia, identificar instrumentos que, de maneira eficiente, auxiliem designers na projeção e mensuração da experiência do usuário, visando o desenvolvimento coerente de produtos e serviços. Neste estudo, devido ao objetivo dos pesquisadores, foi considerado apenas o primeiro nível na avaliação dos instrumentos.

\section{PROCEDIMENTOS METODOLÓGICOS}

Neste artigo, trata-se de relatar o processo (Diagrama 1) e os resultados de uma pesquisa "descritiva", cujos resultados foram organizados de modo comparativo e apresentados em Gráfico 1 e Quadro 1, além de serem descritos em porcentagens. Portanto, os aspectos que primeiramente foram pesquisados de maneira qualitativa, posteriormente também são apresentados quantitativamente. 0 processo inicial do trabalho foi desenvolvido como "pesquisa exploratória", por meio de "revisão bibliográfica sistemática (RBS)" (TRAVASSOS; BIOLCHINI, 2007) em artigos internacionais de língua inglesa, publicados em bases de dados. Os principais termos de busca em língua inglesa foram diretamente relacionados aos significados das expressões portuguesas: "experiência do usuário" e "fatores humanos”. Assim utilizaram-se as palavras-chave: ergonomics ou human factors e user experience. Em seguida fez-se uma pesquisa prévia e definiu-se que as bases de dados consultadas seriam: Web of Science, Scopus e Ebsco. A partir dessas definições, por meio de testes, formatou-se o string de busca:

("ergonom*” OR "human* factor*") AND ("us* experienc*")

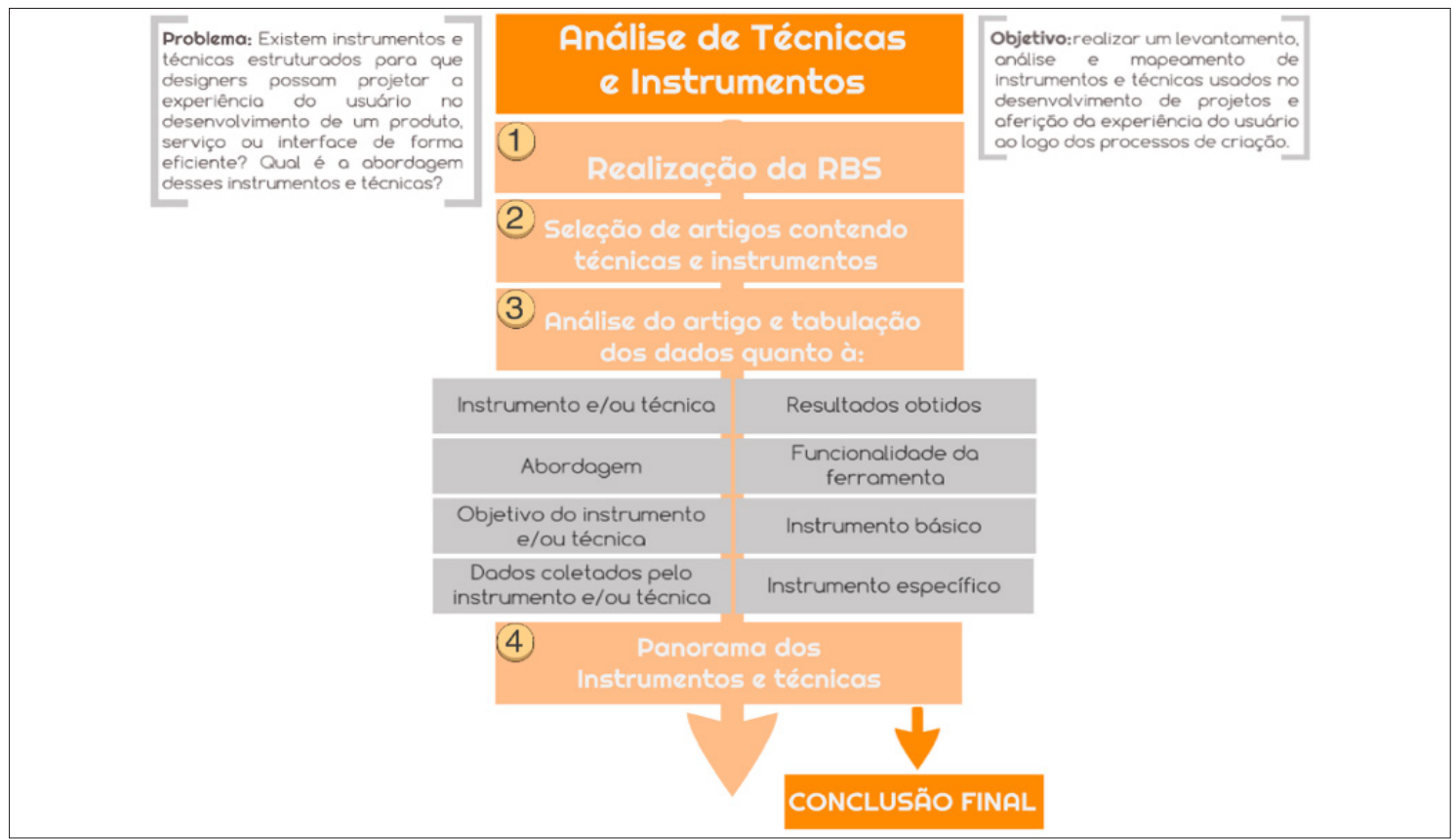

Diagrama 1: Etapas do estudo 
E, assim, definiu-se os critérios de inclusão e de qualificação dos artigos. Foram utilizados como filtro artigos publicados dos anos 2000 a 2017 em inglês. Em um segundo foi feita uma seleção dos artigos por meio dos títulos, depois, em um terceiro momento, resumos e palavras-chaves. Em outra etapa a leitura das introduções e conclusões, para enfim chegar nos artigos selecionados para a leitura e análise completa. Dos artigos analisados procurou-se observar referências, conceitos, ferramentas e métodos utilizados.

Como critérios de inclusão foram selecionados artigos que tratavam sobre fatores humanos/ergonomia e experiência do usuário, levando em conta aspectos subjetivos, hedônicos, percepção de satisfação do usuário e métricas da experiência do usuário.

O instrumentalismo, contudo, foi o foco da pesquisa realizada para identificar os recursos instrumentais já utilizados na mensuração da experiência do usuário. Depois do processo de identificação, houve a breve descrição comparada dos resultados encontrados, caracterizando um processo analítico comparativo. Em síntese: (1) primeiramente, foram identificados nas fontes de pesquisa os instrumentos já utilizados; (2) em seguida foi organizado um panorama descritivo-comparativo do conjunto dos resultados, sendo esse apresentado em gráfico, tabela e texto com porcentagens.

Para este estudo, foram selecionados os artigos que tratavam especificamente de instrumentos de coleta de dados sobre a experiência do usuário. A partir da seleção, as informações foram organizadas em uma tabela, observando algumas características das publicações, como: instrumento e técnicas utilizados; abordagem (qualitativa, quantitativa ou quali-quantitativa); objetivo do instrumento ou técnica; dados coletados; resultados obtidos; funcionalidade; instrumentos básicos e instrumentos específicos. Além disso, no Diagrama 1 são apresentadas as etapas do estudo. Já no Diagrama 2 é apresentada a etapa de seleção dos artigos de forma destrinchada, exibindo o resultado de artigos obtidos em cada filtro até a obtenção do portfólio final de 45 artigos analisados neste estudo.

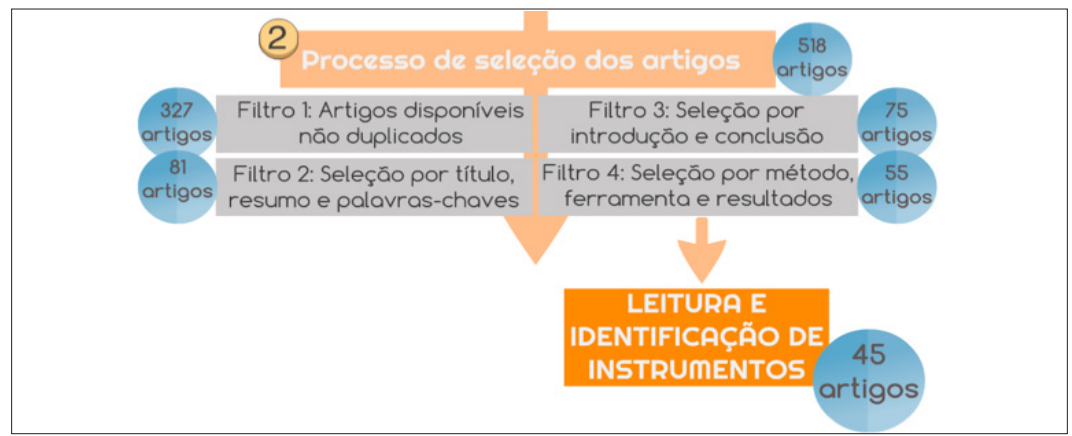

\section{RESULTADOS}

\section{Instrumentos e técnicas de mensuração da experiência do usuário}

A partir da análise dos 55 artigos selecionados no processo de revisão bibliográfica sistemática, foram selecionados aqueles que traziam algum tipo de instrumento ou técnica de mensuração da experiência do usuário. Foram selecionados 45 artigos publicados no período de 2000 a 2017 e que apresentaram algum instrumento ou técnica de mensuração para compor o portfólio final para a análise. Dentro dos limites da pesquisa realizada, devido ao maior número de artigos publicados, foi considerado que houve um aumento exponencial do interesse pelo tema no ano de 2007. Portanto, houve um período de crescimento, relativamente pequeno e recente, a partir desse, ocorrendo um pico de crescimento no ano de 2016, levando em consideração que época limite da realização da pesquisa realizada foi o primeiro semestre de 2017 (Gráfico 1).
Diagrama 2: Resultados das buscas e filtros

Fonte: Elaborado pelos autores 


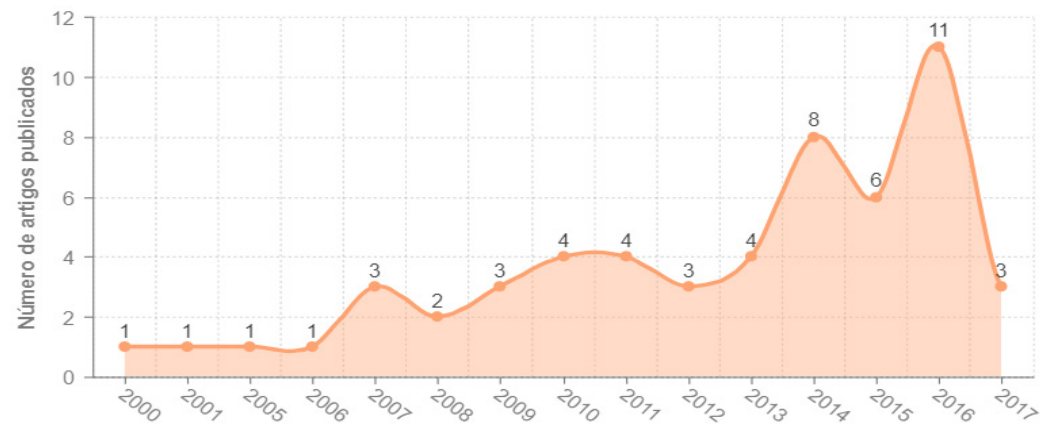

Fonte: elaborado pelos autores

Para realizar a análise do portfólio de artigos, os dados coletados em cada uma das publicações foram inclusos, separados e categorizados por meio de uma planilha do programa Microsoft Office Excel. Criou-se um quadro contendo as seguintes características de cada artigo:

- metodologia ou instrumento (ferramenta) utilizada;

- tipo de abordagem;

- objetivo do instrumento ou método;

- dados coletados;

- resultados obtidos;

- se o objetivo do instrumento foi alcançado;

- ferramentas básicas (instrumentos com características generalistas que são frequentemente encontrados em estudos científicos, como: questionários, entrevistas, observação e focus group); e

- ferramentas específicas (instrumentos com características específicas dos estudos ou desenvolvidos para fins exclusivos, usados para coletar dados peculiares pertinentes ao tema deste artigo).

Dessa forma, criou-se o Quadro 1 apresentado a seguir. Nele são apresentados esquematicamente os dados comparativos dos instrumentos pesquisados nos 45 artigos selecionados.

\section{Análise do Quadro 1 - avaliação de funcionalidade de instrumentos e técnicas}

Com base no Quadro 1 podemos observar que, dos 45 artigos avaliados, a maioria trata da experiência do usuário voltada para interfaces digitais, representando $47 \%$ dos artigos; $24 \%$ investigam a experiência do usuário voltada para produtos; $5 \%$, para serviços; e $9 \%$ dos artigos são voltados para metodologias de projetos. Outros artigos traziam a combinação de mais de um domínio: $9 \%$ sobre interface digital e produto, $2 \%$ tratavam de interface digital e serviço, $2 \%$ sobre produto e serviço e $2 \%$ a respeito de metodologia de projeto e interface digital.

Os instrumentos e as técnicas identificados nos artigos foram avaliados conforme o primeiro nível de Feenberg (2010): cumprimento da função designada ou sua funcionalidade. Para isso, a partir desses instrumentos e técnicas levantados, buscou-se avaliar os objetivos de cada um desses, os dados coletados, os resultados obtidos, cruzados com as conclusões dos autores de cada artigo. Dessa forma, observou-se que $9 \%$ dos artigos não obtiveram os resultados esperados ou adotaram as técnicas satisfatórias para cumprir com os objetivos propostos, o que corresponde a quatro artigos. Por outro lado, 91\% dos estudos utilizaram de instrumentos e técnicas que cumpriram com sua função. 


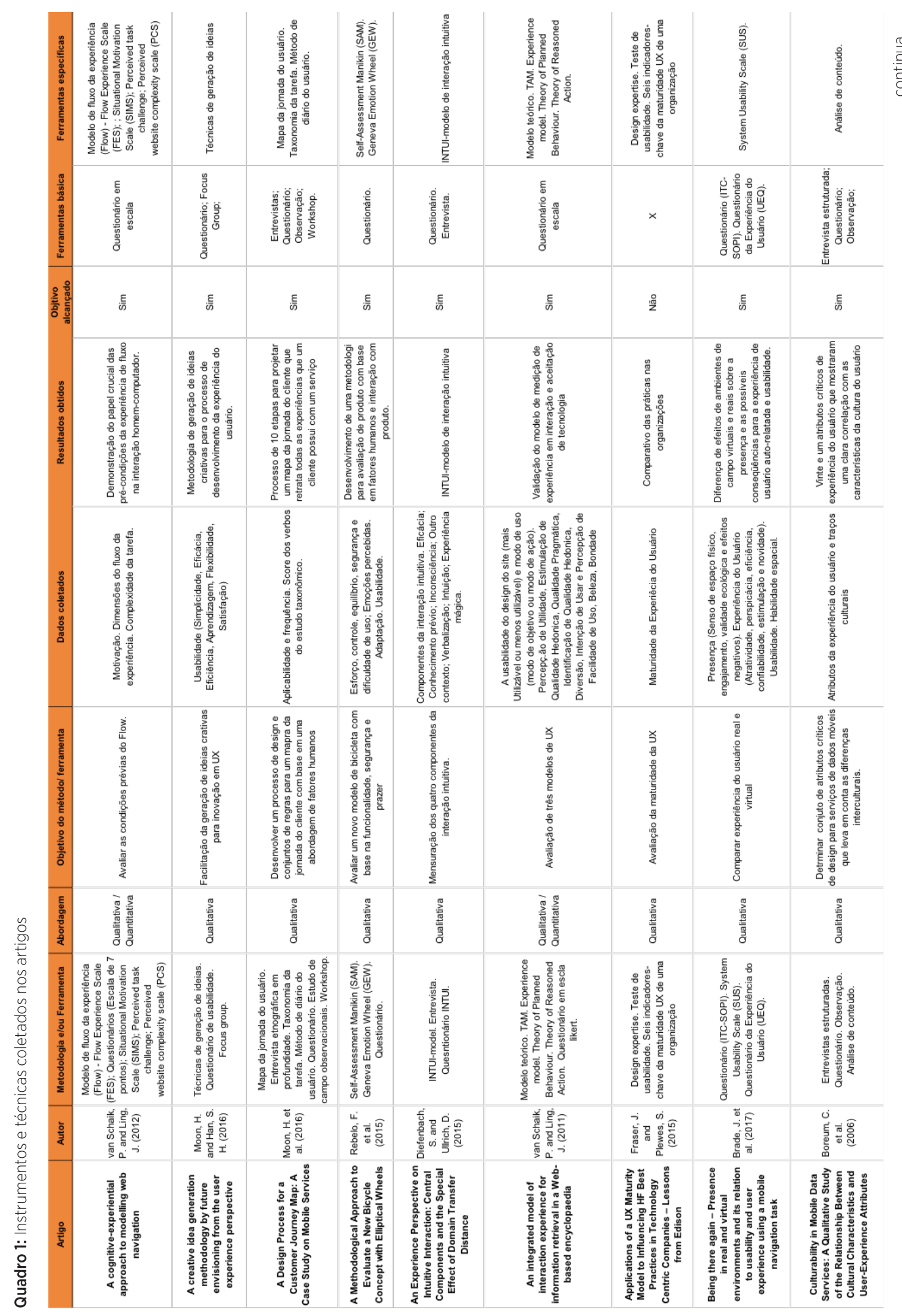




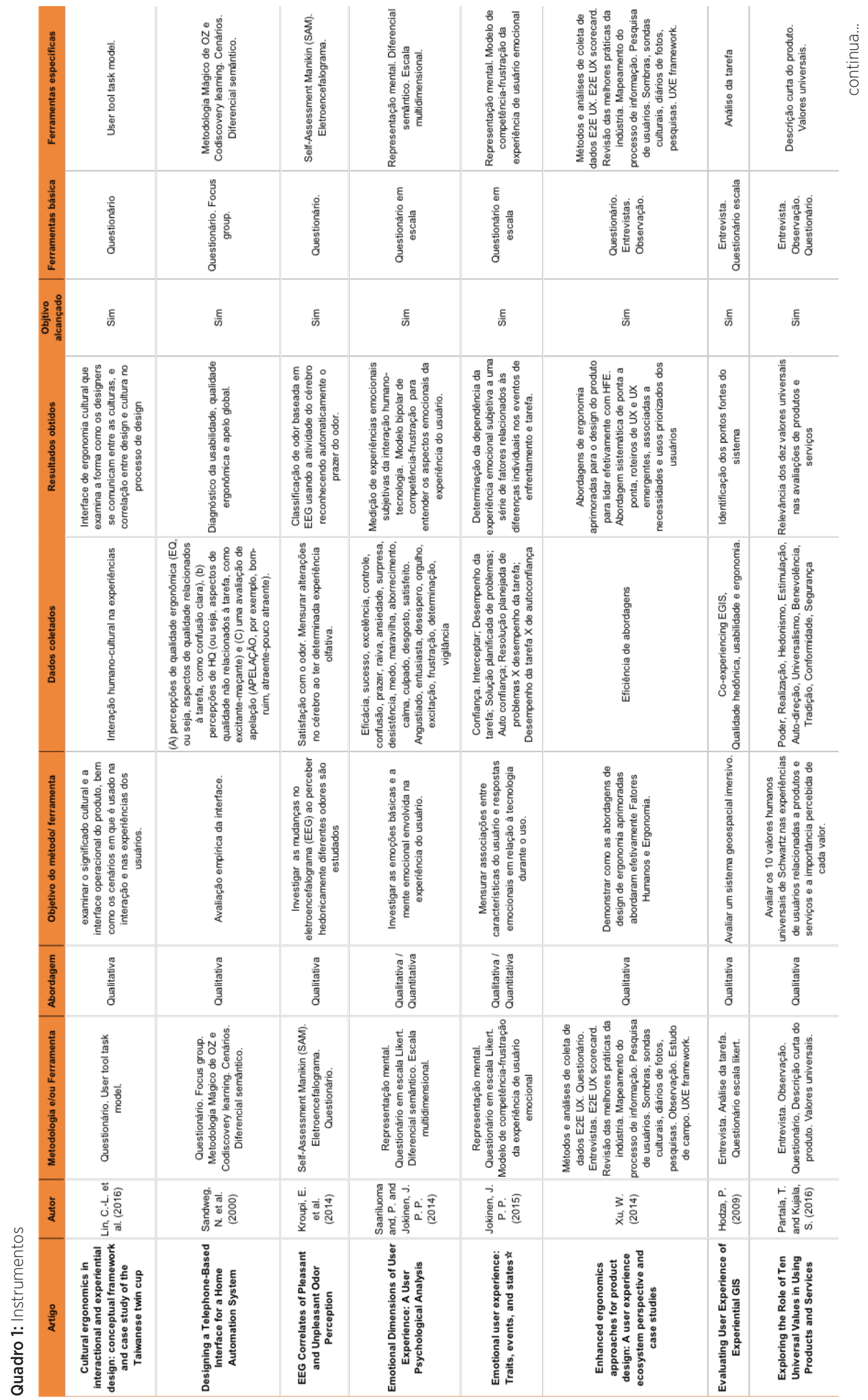




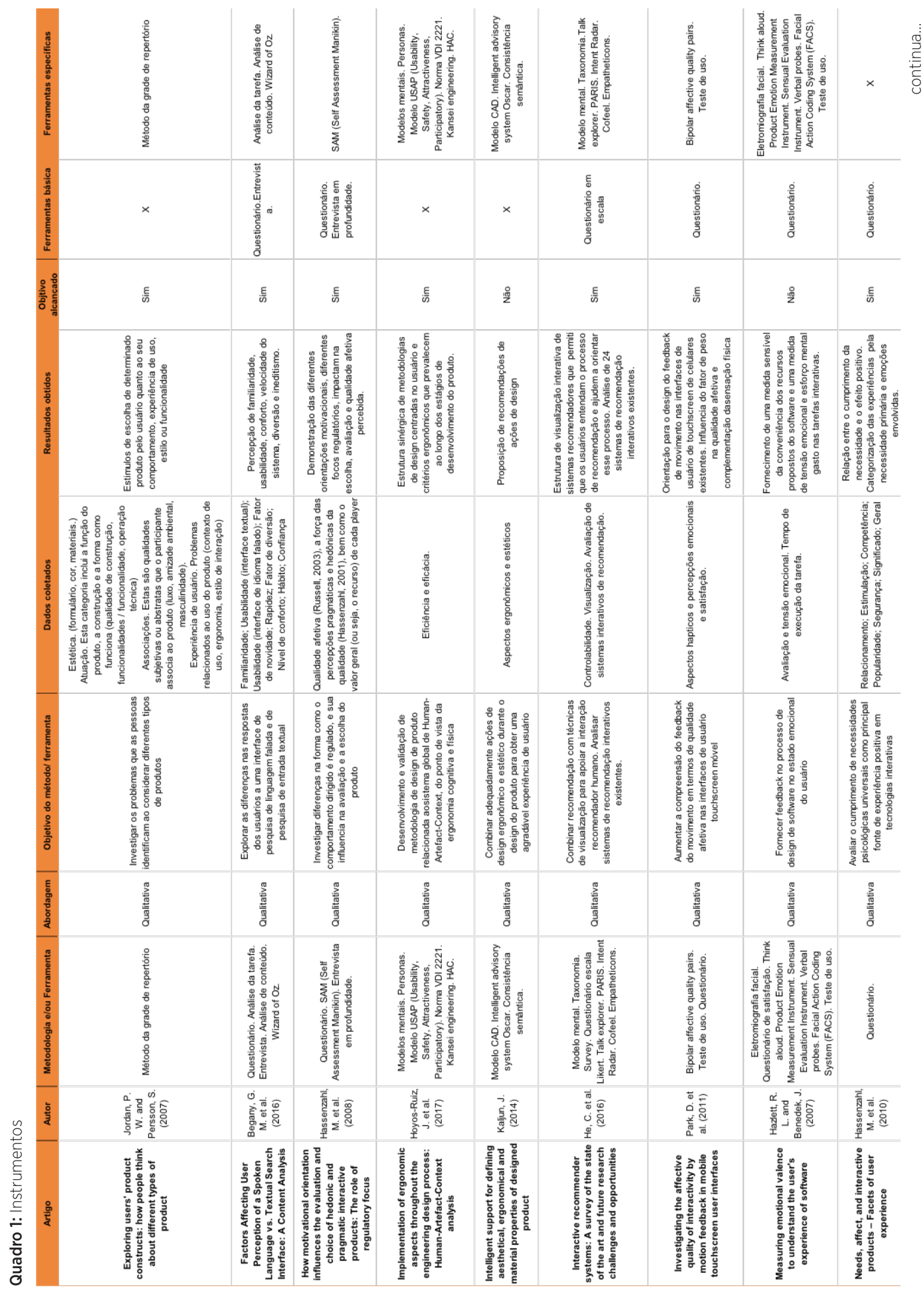




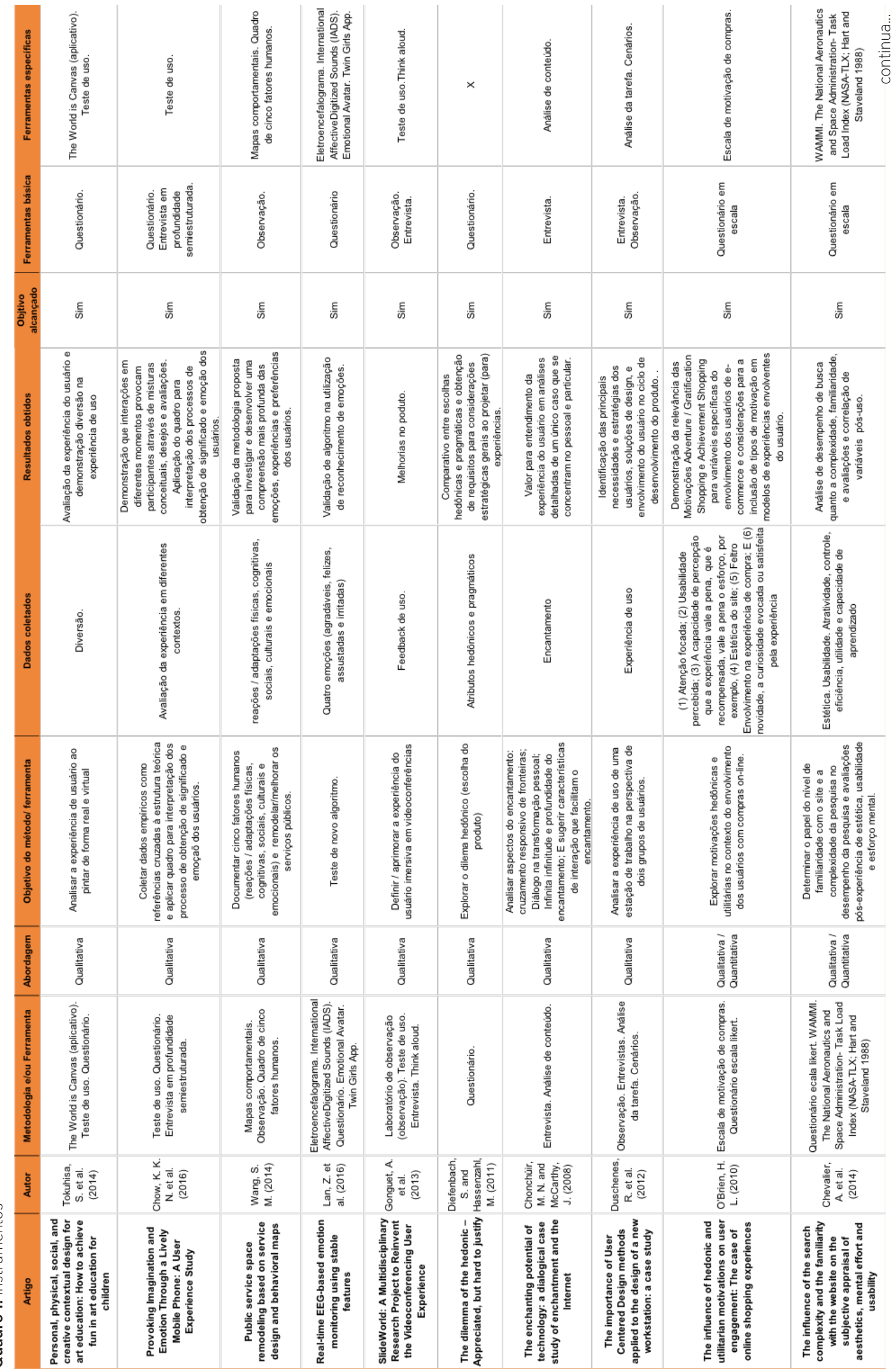

44 Gestão e Tecnologia de Projetos 


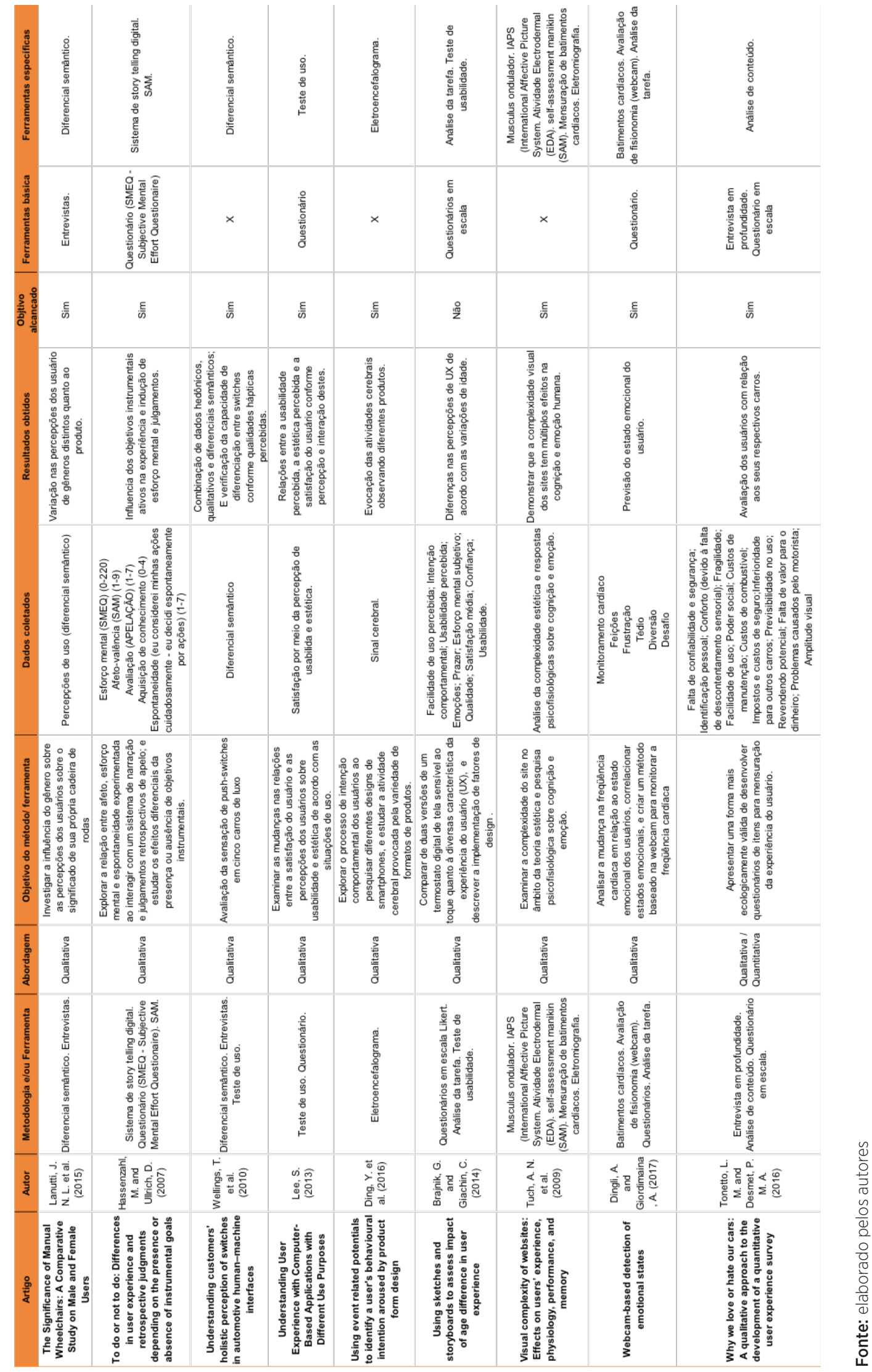


Figura 1: Gráficos com análise de instrumentos e técnicas

Fonte: Elaborado pelos autores
Na Figura 1 é apresentada graficamente a síntese dos dados coletados e analisados no Quadro 1.
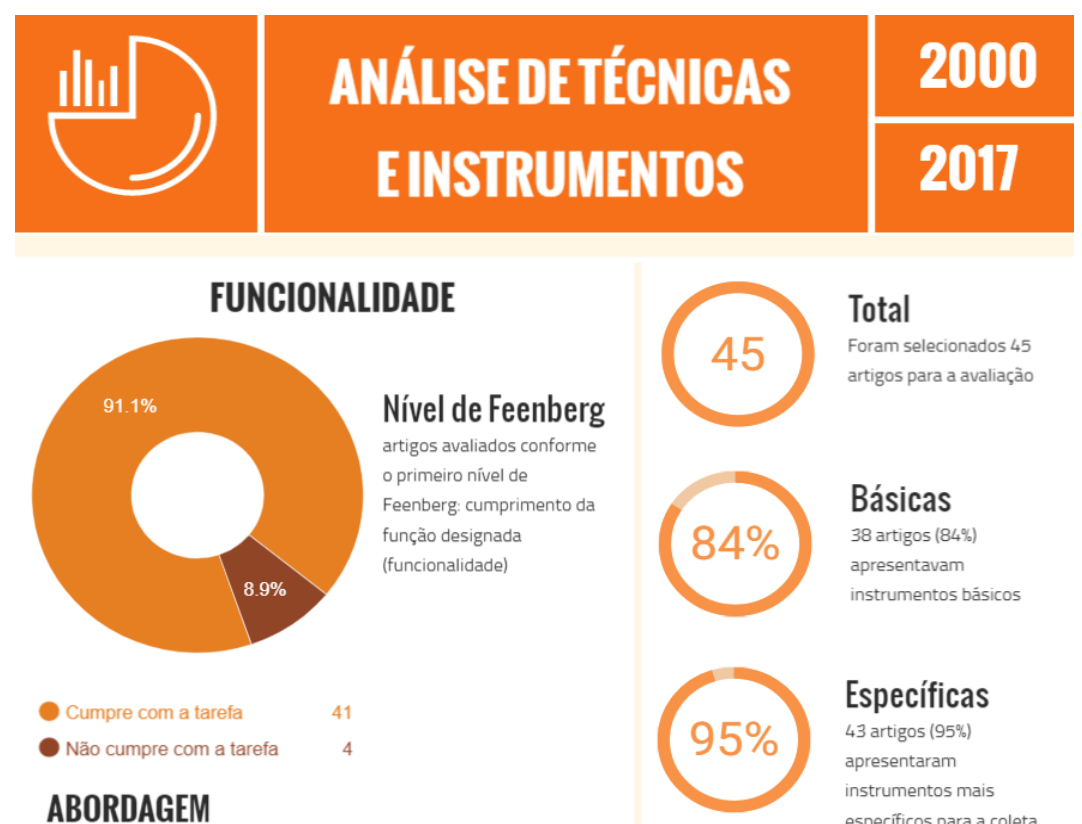

\section{Específicas}

43 artigos (95\%) apresentaram instrumentos mais especificos para a coleta

\section{ABORDAGEM}

$84,4 \%$ dos artigos tiveram abordagem qualitativa, enquanto $15,6 \%$ trabalharam com abordagem qualitativa, porém traduzindo-os em dados quantitativos. Nenhum artigo teve abordagem apenas quantitativa
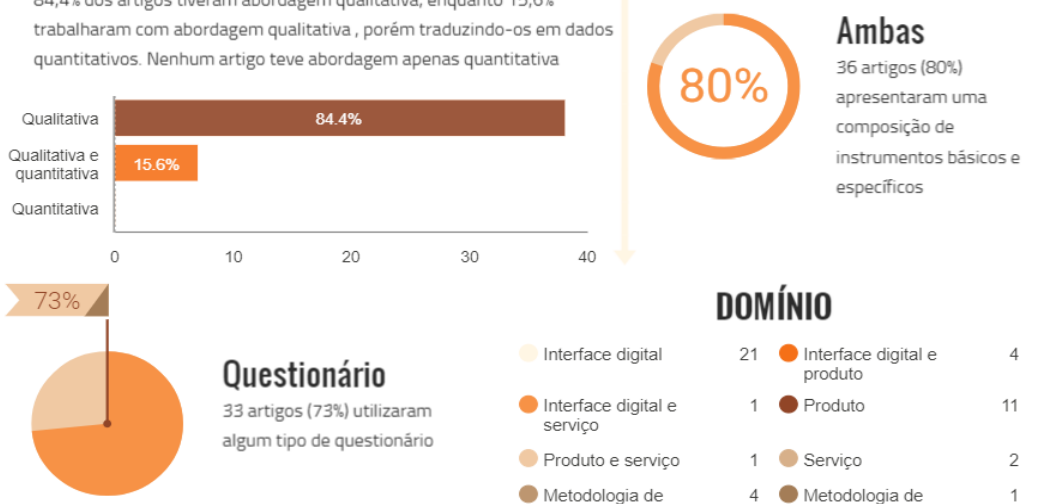

33 artigos (73\%) utilizaram algum tipo de questionário

\section{Entrevista}

14 artigos (37\%) utilizaram algum tipo de entrevista

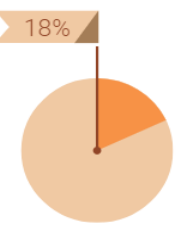

\section{DOMÍNIO}

\begin{tabular}{|c|c|c|c|}
\hline \\
\hline Interface digital & 21 & $\begin{array}{l}\text { Interface digital e } \\
\text { produto }\end{array}$ & 4 \\
\hline $\begin{array}{l}\text { Interface digital e } \\
\text { serviço }\end{array}$ & 1 & Produto & 11 \\
\hline Produto e serviço & 1 & Serviço & 2 \\
\hline $\begin{array}{l}\text { Metodologia de } \\
\text { projeto }\end{array}$ & 4 & $\begin{array}{l}\text { Metodologia de } \\
\text { projeto e interface } \\
\text { digital }\end{array}$ & 1 \\
\hline
\end{tabular}

\section{Observação}

7 artigos (18\%) utilizaram observação

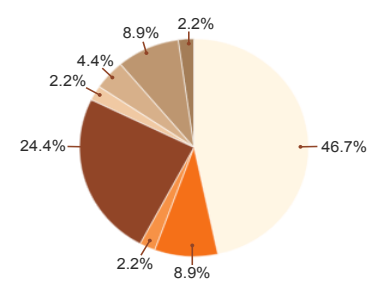

21 artigos tratam a UX voltada para interfaces digitais $(46,7 \%) .11$ artigos tratam da UX voltada para produtos $(24,4 \%)$, e 2 artigos para serviços (5\%)

\section{Abordagem}

Quanto à abordagem (Quadro 1), e como observado na Figura 1, nenhum dos artigos assumiu abordagem exclusivamente quantitativa. Isso poderia ser justificado devido à experiência do usuário possuir caráter subjetivo, isto é, ser composta por elementos efêmeros e subjetivos (HASSENZAHL; DIEFENBACH; GÖRITZ, 2010; LAW et al., 2009). 84,4\% dos artigos adotaram uma abordagem 
apenas qualitativa, enquanto 15,6\% das publicações buscaram trabalhar com abordagem qualitativa, porém traduzi-los em dados quantitativos, tendo uma abordagem quanti-qualitativa.

\section{Tipos de instrumentos de coleta observados}

A partir do Quadro 1 gerado, foi possível obter uma listagem de instrumentos (ferramentas básicas e específicas) e assim pode-se fazer a correspondência dos objetivos dos estudos, dados coletados e instrumentos adotados. Para classificação dos instrumentos e técnicas de coleta utilizados nos artigos, dividiu-se em dois tipos: os instrumentos mais usuais (questionário, entrevista, observação e análise de conteúdo) (MARCONI; LAKATOS, 2008), aqui chamados de instrumentos básicos; e os instrumentos específicos usados para coletar e avaliar dados particulares aos estudos e que podem combinar diversas formas de medições e equipamentos. Dos instrumentos observados nos estudos, 38 artigos apresentaram instrumentos básicos, o que corresponde a $84 \%$ do total. Já instrumentos específicos para a coleta de dados particulares à temática de cada pesquisa apareceram em 43 artigos, representando 95\% dos 45 artigos. E, por fim, 36 artigos apresentaram ambos os tipos (básico e específico), totalizando 80\% dos estudos.

Quanto aos instrumentos básicos, 73\% dos artigos aplicaram algum tipo de questionário, 37\% fizeram algum tipo de entrevista e 18\% dos estudos utilizaram observação. Nenhum dos artigos analisados utilizou alguma forma de análise de conteúdo como método de coleta. Apesar de todos os artigos adotarem abordagem qualitativa, apenas dois deles utilizaram instrumentos básicos para suas coletas, não adotando instrumentos específicos. Em ambos os artigos, os autores propuseram como atividades futuras a combinação ou aplicação de novas técnicas para a obtenção de dados mais precisos ou validação dos resultados.

Gráfico 2: Questionários com escala Likert

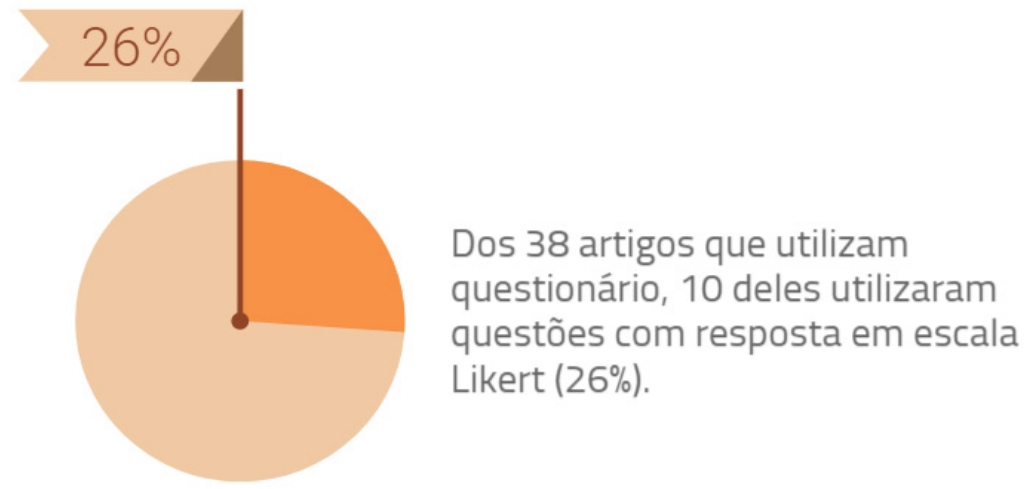

Fonte: elaborado pelos autores

Os artigos que tiveram uma abordagem quanti-qualitativa, em um primeiro momento, coletaram os dados qualitativos. Contudo utilizaram questões em escala Likert para que assim, posteriormente, fosse possível converter os dados coletados em scores e a partir deles realizar testes estatísticos, como análise fatorial, e atribuir caráter quantitativo. Dos 38 artigos que utilizaram alguma forma de questionário em sua coleta, 10 utilizaram a escala Likert em suas respostas, totalizando $26 \%$ das publicações (Gráfico 2). 


\section{CONSIDERAÇÕES FINAIS}

O design voltado para a projetação da experiência do usuário é uma temática recente, com instrumentos e técnicas ainda não bem definidos. Assim existe a dificuldade de se determinar os fatores humanos que influenciam nessa experiência, bem como obter resultados concretos que delimitem os aspectos que compõem a experiência e o valor atribuído a ela pelo usuário. Por isso a importância de se investigar e refletir sobre a origem do termo, considerando também as linhas de pensamentos que, tradicionalmente, valorizaram a experiência do indivíduo e a percepção dos recursos científicos como instrumentos do conhecimento, como foi proposto nas ideias de John Dewey.

Para ampliar os conhecimentos sobre a experiência do usuário, incrementando o embasamento teórico e prático de design na projetação de produtos, serviços ou interfaces, o interesse deste artigo foi identificar instrumentos que, de maneira eficiente, auxiliam designers na projeção e mensuração da experiência do usuário, visando o desenvolvimento coerente de produtos e serviços. Para tanto, realizou-se um estudo bibliométrico das publicações que, mais especificamente, buscaram as relações já estabelecidas entre experiência do usuário e fatores humanos/ergonomia, resultando em uma análise quantitativa e qualitativa dos dados. Tal pesquisa foi realizada considerando o contexto teórico da doutrina designada como instrumentalismo e, por isso, houve o interesse pela pesquisa de instrumentos e técnicas nos artigos selecionados.

A adoção do sistema de classificação de Feenberg (2010) serviu para aprofundar o estudo das publicações, observando a funcionalidade dos instrumentos e sua efetividade de acordo com os resultados propostos em cada artigo pesquisado. Possibilitou assim a construção de um quadro (Quadro 1) que condensasse as informações colhidas com base no portfólio de 45 artigos resultantes da revisão sistemática. Esse quadro permitiu a visualização das informações de forma holística e, a partir disso, apresentou um panorama de técnicas e instrumentos relacionados com experiência do usuário e fatores humanos/ergonomia, considerando ainda as possibilidades futuras de desenvolvimento de novos recursos e metodologias. Isso foi especialmente cogitado porque observou-se que não existe um padrão evidente nas técnicas utilizadas para a aferição da experiência do usuário (UX).

Diante dos resultados da pesquisa realizada, considerou-se que os processos quantitativos, a partir de observações ou estudos preliminares qualitativos, buscaram propor métricas ou valores mais precisos e replicáveis, com a aplicação de instrumentos objetivos para o registro das impressões dos usuários, como a escala Likert e testes estatísticos. Também foi considerado o uso de recursos tecnológicos para o monitoramento das reações fisiológicas dos usuários, como eletroencefalograma e mapas de frequência cardíaca, entre outros. Avaliando-se o necessário interesse por dados quantitativos no campo tecnológico de Ciência Aplicada, observou-se também a necessidade de adoção ou desenvolvimento de instrumentos métricos para reconhecimento, registro e entendimento sobre as experiências dos usuários, visando aprimorar cada vez mais a precisão e a validação das informações e, principalmente, a aplicação desse conhecimento em melhores produtos, interfaces ou serviços.

Acompanhando esse interesse de adoção de instrumentos métricos e reconhecimento das experiências, percebe-se uma tendência, nos estudos analisados, de adoção de ferramentas específicas para essa mensuração, ou até mesmo a combinação de duas ou mais ferramentas de coleta (básicas e específicas) para o alcance dos objetivos de cada publicação. Apenas dois artigos não apresentaram ferramentas específicas para coletar e aferir a experiência do usuário. Portanto, dada a complexidade das experiências dos usuários, fica perceptível a necessidade dos pesquisadores em utilizar mais de um instrumento. Ferramentas básicas mostram-se mais generalistas, podendo auxiliar o pesquisador na delimitação do perfil do usuário, percepções e observações particulares e qualitativas do indivíduo no contexto da UX. Já as ferramentas específicas podem auxiliar na coleta de aspectos peculiares 
da experiência, ajudando o pesquisador a analisar particularidades que compõem a experiência em questão. Por isso, observa-se como possível lacuna o desenvolvimento de uma ferramenta voltada para aferição da experiência do usuário que pondere características de ferramentas básicas e específicas.

Como limitações deste trabalho apontam-se: (1) pesquisa bibliográfica restrita a artigos escritos em língua inglesa, publicados em revistas científicas indexadas nas bases de dados disponíveis no portal de periódicos da Capes e disponíveis gratuitamente na internet; (2) análise das características definidas pelos pesquisadores que possibilitaram a reflexão e identificação de oportunidades de futuras pesquisas; (3) adoção de apenas o primeiro nível de análise proposto por Feenberg (2010); e (4) análise dos artigos do portfólio bibliográfico em relação às variáveis investigadas de acordo com o julgamento e a interpretação dos autores desta pesquisa, por isso, podendo não corresponder às conclusões dos autores originais dos estudos. E por fim, sugere-se para trabalhos futuros a ampliação deste estudo a outras bases de dados e o desenvolvimento de trabalhos que visem eliminar as lacunas apontadas ao longo do artigo.

\section{AGRADECIMENTOS}

Agradecemos à Coordenação de Aperfeiçoamento de Pessoal de Nível Superior (Capes), à Rede de Pesquisa e Desenvolvimento de Tecnologia Assistiva (RPDTA), ao Programa de Pós-graduação em Design da Universidade Federal de Santa Catarina (PPGD/UFSC) e ao Núcleo de Gestão de Design e Laboratório de Design e Usabilidade (NGD-LDU/UFSC).

\section{REFERÊNCIAS}

AZEVEDO, W. O que é design. 3. ed. São Paulo: Brasiliense, 2006

BEGANY, G. M.; NING, S. A.; XIAOJUN, Y. Factors affecting user perception of a spoken language vs. textual search interface: a content analysis. Interacting with Computers, Oxford, v. 28, n. 2, p. 170-180, 2016.

BOMFIM, G. A. Fundamentos de uma teoria transdisciplinar do design: morfologia dos objetos de uso e sistema de comunicação. Estudos em Design, Rio de Janeiro, v. 5, n. 2, p. 27-41, 1997.

BONSIEPE, G. Design como prática de projeto. São Paulo: Blucher, 2012.

BOREUM, C.; INSEONG, L.; JINWOO, K. Culturability in mobile data services: a qualitative study of the relationship between cultural characteristics and user-experience attributes. International Journal of HumanComputer Interaction, Abing don, v. 20, n. 3, p. 171-203, 2006.

BRADE, J. et al. Being there again - presence in real and virtual environments and its relation to usability and user experience using a mobile navigation task. International Journal of Human-Computer Studies, Amsterdam, v. 101, p. 76-87, 2017.

BRAJNIK, G.; GIACHIN, C. Using sketches and storyboards to assess impact of age difference in user experience. International
Journal of Human-Computer Studies Amsterdam, v. 72, n. 6, p. 552-566, 2014.

BUNGE, M. La investigación científica. Barcelona: Ariel, 1969.

Treatise on basic philosophy. Dordrecht: Reidel, 1985. v. 7.

CAMPOS, J. et al. Aspectos da filosofia de Immanuel Kant aplicados ao design. Triades. Rio de Janeiro, v. 4, n. 1, 2015.

CHEVALIER, A.; MAURY, A. C.; FOUQUEREAU, $\mathrm{N}$. The influence of the search complexity and the familiarity with the website on the subjective appraisal of aesthetics, mental effort and usability. Behaviour and Information Technology, Abingdon, v. 33, n. 2, p. 116-131, 2014.

CHONCHÚIR, M. N.; MCCARTHY, J. The enchanting potential of technology: a dialogical case study of enchantment and the Internet. Personal \& Ubiquitous Computing, New York, v. 12, n. 5, p. 401-409, 2008.

CHOW, K. K. et al. Provoking imagination and emotion through a lively mobile phone: a user experience study. Interacting with Computers, Oxford, v. 28, n. 4, p. 451-461, 2016.

CUPANI, A. A tecnologia como problema filosófico: três enfoques. Scientiae Studia, São Paulo, v. 2, n. 4, p. 493-518, 2004. 
DEWEY, J. Como pensamos. São Paulo: Companhia Editora Nacional, 1959.

Vida e educação. Tradução e estudo preliminar por Anísio S. Teixeira. São Paulo: Melhoramentos; Rio de Janeiro: Fundação Nacional de Material Escolar, 1978.

. O desenvolvimento do Pragmatismo Americano. Cognitio-Estudos, São Paulo, v. 5, n. 2, p. 119-132, 2008.

DIEFENBACH, S.; HASSENZAHL, M. The dilemma of the hedonic: appreciated, but hard to justify. Interacting with Computers, Oxford, v. 23, n. 5, p. 461-472, 2011.

DIEFENBACH, S.; ULLRICH, D. An experience perspective on intuitive interaction: central components and the special effect of domain transfer distance. Interacting with Computers, Oxford, v. 27, n. 3, p. 210-234, 2015.

DING, Y. et al. Using event related potentials to identify a user's behavioural intention aroused by product form design. Applied Ergonomics, Amsterdam, v. 55, p. 117-123, 2016.

DINGLI, A.; GIORDIMAINA, A. Webcambased detection of emotional states. Visual Computer, New York, v. 33, n. 4, p. 459-469, 2017.

DUSCHENES, R. et al. The importance of user centered design methods applied to the design of a new workstation: a case study. Work-a Journal of Prevention Assessment \& Rehabilitation, Amsterdam, v. 41, p. 984988,2012

DWEK, M; COUTINHO, H; MATHEUS, F. Por uma formação crítica em engenharia. In: CONGRESSO BRASILEIRO DE EDUCAÇÂO EM ENGENHARIA, 39., 3-6 out. 2011, Blumenau. Anais... Brasília, DF: Abenge, 2011.

FEENBERG, A. Teoria crítica da tecnologia: um panorama. In: NEDER, R. T. (Org.). Andrew Feenberg: racionalização democrática, poder e tecnologia. Brasília, DF: Observatório do Movimento pela Tecnologia Social na América Latina/Centro de Desenvolvimento Sustentável. (Série Cadernos Primeira Versão: CCTS - Construção Crítica da Tecnologia \& Sustentabilidade, 2010 v. 1., n. 3)

FERREIRA, N. G. M. L. O papel da experiência na filosofia de John Dewey. Filogenese, Marília, v. 4, n. 2, p. 147.156, 2011.

FRASER, J.; PLEWES, S. Applications of a UX maturity model to influencing HF best practices in technology centric companies: lessons from Edison. Procedia Manufacturing, Amsterdam, v. 3, p. 626-631, 2015.

GONGUET, A. et al. SlideWorld: a multidisciplinary research project to reinvent the videoconferencing user experience. Bell Labs Technical Journal, Hoboken, v. 17, n. 4, p. 133-144, 2013.

GREEN, W.; JORDAN, P. W. (Ed.). Human factors in product design: current practice and future trends. Abingdon: CRC Press, 1999.

HASSENZAHL, M. The interplay of beauty, goodness, and usability in interactive products. Human-Computer Interaction, Abingdon, v. 19, n. 4, p. 319-349, 2004.

Experience design: technology for all the right reasons. London: Morgan \& Claypool Publishers, 2010.

HASSENZAHL, M.; ULLRICH, D. To do or not to do: differences in user experience and retrospective judgments depending on the presence or absence of instrumental goals. Interacting with Computers, Oxford, v. 19, n. 4, p. 429-437, 2007

HASSENZAHL, M.; SCHÖBEL, M.; TRAUTMANN, T. How motivational orientation influences the evaluation and choice of hedonic and pragmatic interactive products: the role of regulatory focus. Interacting with Computers, Oxford, v. 20, n. 4-5, p. 473-479, 2008.

HASSENZAHL, M.; DIEFENBACH, S.; GÖRITZ, A. Needs, affect, and interactive products: facets of user experience. Interacting with Computers, Oxford, v. 22, n. 5, p. 353-362, 2010.

HAZLETT, R. L.; BENEDEK, J. Measuring emotional valence to understand the user's experience of software. International Journal of Human-Computer Studies, Amsterdam, v. 65, n. 4, p. 306-314, 2007

HE, C.; PARRA, D.; VERBERT, K. Interactive recommender systems: a survey of the state of the art and future research challenges and opportunities. Expert Systems with Applications, Amsterdam, v. 56, p. 9-27, 2016.

HODZA, P. Evaluating user experience of experiential GIS. Transactions in GIS, Hoboken, v. 13, n. 5-6, p. 503-525, 2009.

HOYOS-RUIZ, J. et al. Implementation of ergonomic aspects throughout the engineering design process: human-artefactcontext analysis. International Journal on Interactive Design and Manufacturing. New York, v. 11, n. 2, p. 263-277, 2017.

IIDA, I.; BUARQUE, L. Ergonomia: projeto e produção. 3. ed. São Paulo: Blucher, 2016.

JAPIASSÚ, H.; MARCONDES, D. Dicionário básico de filosofia. 3. ed. Rio de Janeiro: Zahar, 1996

JOKINEN, J. P. P. Emotional user experience: traits, events, and states. International 
Journal of Human-Computer Studies Amsterdam, v. 76, p. 67-77, 2015.

JORDAN, P. W.; PERSSON, S. Exploring users' product constructs: how people think about different types of product. CoDesign Abingdon, v. 3, p. 97-106, 2007.

KALJUN, J. Intelligent support for defining aesthetical, ergonomical and materia properties of designed product. Tehničk Vjesnik, Slavonski Brod, v. 21, n. 4, p. 835842, 2014

KOTLER, P.; KELLER, K. L. Administração de marketing. 12. ed. São Paulo: Pearson Prentice Hall, 2006

KROUPI, E. et al. EEG correlates of pleasant and unpleasant odor perception. ACM Transactions on Multimedia Computing Communications and Applications, NeW York, v. 11, n. 1, p. 13, 2014

LAN, Z. et al. Real-time EEG-based emotion monitoring using stable features. The Visual Computer, New York, v. 32, n. 3, p. 347-358, 2016.

LANUTTI, J. N. L. et al. The significance of manual wheelchairs: a comparative study on male and female users. Procedia Manufacturing, Amsterdam, v. 3, p. 6079 6085, 2015.

LAURANS, G. F. G.; DESMET, P. M. A.; HEKKERT, P. Assessing emotion in interaction: some problems and a new approach In: INTERNATIONAL CONFERENCE ON DESIGNING PLEASURABLE PRODUCTS AND INTERFACES, 4., 13-16 out. 2009, Compiègne. Proceedings... Compiegne: UTC, 2009.

LAW, E. L.-C.; ROTO, V.; HASSENZAHL, M. VERMEEREN, A; KORT, J. Understanding, scoping and defining user experience: a survey approach. In: INTERNATIONAL CONFERENCE ON HUMAN FACTORS IN COMPUTING SYSTEMS, 27., 2009. Proceedings... Nova York: ACM Press, 2009. p. 719-728.

LEE, S. Understanding user experience with computer-based applications with different use purposes. International Journal of Human-Computer Interaction, Abingdon, v. 29, n. 11, p. 689-701, 2013.

LIN, C. L. et al. Cultural ergonomics in interactional and experiential design: conceptual framework and case study of the Taiwanese twin cup. Applied Ergonomics, Amsterdam, v. 52, p. 242-252, 2016

LÖBACH, B. Design industrial: bases para a configuração dos produtos industriais. São Paulo: Blucher, 2001

LORIERI, M. A. Aspectos do instrumentalismo pragmatista na teoria do conhecimento de John Dewey. Cognitio-Estudos, São Paulo, n. 1, p. 46-57, 2000
MARCONI, M. A.; LAKATOS, E. M. Metodologia

científica. 5. ed. São Paulo: Atlas, 2008.

MOON, H.; HAN, S. H. A creative idea generation methodology by future envisioning from the user experience perspective. International Journal of Industrial Ergonomics, Amsterdam, v. 56, p. 84-96, 2016.

MOON, H. et al. A design process for a customer journey map: a case study on mobile services. Human Factors and Ergonomics in Manufacturing \& Service Industries Hoboken, v. 26, n. 4, p. 501-514, 2016

O'BRIEN, H. L. The influence of hedonic and utilitarian motivations on user engagement: the case of online shopping experiences. Interacting with Computers, Oxford, v. 22, n. 5, p. 344-352, 2010.

OLIVEIRA, R. N; LIMEIRA, C. D; SANTAROSA, J. G. A experiência do usuário no processo evolutivo do design. Blucher Design Proceedings, São Paulo, v. 1, n. 4, p. 34513460, 2014.

PANTALEÃO, L. F; PINHEIRO, O. J. A intuição e o acaso no processo criativo: questões de metodologia para a inovação em design. In: CONGRESSO INTERNACIONAL DE PESQUISA EM DESIGN, 5., 10-12 out. 2009, Bauru. Anais.. São Paulo: Anped, 2009.

PARK, D.; LEE, J. H.; KIM, S. Investigating the affective quality of interactivity by motion feedback in mobile touchscreen user interfaces. International Journal of HumanComputer Studies, Amsterdam, v. 69, n. 12, p. 839-853, 2011.

PARTALA, T.; KUJALA, S. Exploring the role of ten universal values in using products and services. Interacting with Computers Oxford, v. 28, n. 3, p. 311-331, 2016

POELS, K.; DEWITTE, S. How to capture the heart? Reviewing 20 years of emotion measurement in advertising. Journal of Advertising Research, Abingdon, v. 46 , n. 1, p. 18-37, 2006.

REBELO, F. et al. A methodological approach to evaluate a new bicycle concept with elliptical wheels. Procedia Manufacturing, Amsterdam, v. 3, p. 6361-6368, 2015.

SAARILUOMAAND, P.; JOKINEN, J. P. P. Emotional dimensions of user experience: a user psychological analysis. International Journal of Human-Computer Interaction, Abingdon, v. 30, n. 4, p. 303-320, 2014.

SANDWEG, N.; HASSENZAHL, M.; KUHN, K. Designing a telephone-based interface for a home automation system. International Journal of Human-Computer Interaction, Abingdon, v. 12, n. 3-4, p. 401-414, 2000 
SANTOS, M. C. F. A noção de experiência em John Dewey, a educação progressiva e o currículo de ciências. In: ENCONTRO NACIONAL DE PESQUISA EM EDUCAÇÃO E CIÊNCIAS, 8., 5-9 dez. 2011, Campinas. Atas... São Paulo: Abrapec, 2012. Disponível em: <https://goo.gl/E6MELc>. Acesso em: 17 jun. 2017

TRAVASSOS, G.; BIOLCHINI, J. Revisões sistemáticas aplicadas a engenharia de software. In: SBES-BRAZILIAN SYMPOSIUM ON SOFTWARE ENGINEERING, 21, 2007. Anais...

TOKUHISA, S.; KAMIYAMA, Y.; TOKIWA, T. Personal, physical, social, and creative contextual design for art education: How to achieve fun in art education for children. Computers in Entertainment, New York, v. 11, n. 4, p. 2, 2014.

TONETTO, L. M.; DESMET, P. M. A. Why we love or hate our cars: a qualitative approach to the development of a quantitative user experience survey. Applied Ergonomics, Amsterdam, v. 56, p. 68-74, 2016.

TUCH, A. N. et al. Visual complexity of websites: effects on users' experience, physiology, performance, and memory.
International Journal of Human-Computer Studies, Amsterdam, v. 67, n. 9, p. 703-715, 2009 .

VAN SCHAIK, P.; LING, J. An integrated model of interaction experience for information retrieval in a Web-based encyclopaedia. Interacting with Computers, Oxford, v. 23, n. 1, p. 18-32, 2011.

. A cognitive-experiential approach to modelling web navigation. International Journal of Human-Computer Studies, Amsterdam, v. 70, n. 9, p. 630-651, 2012.

WANG, S. M. Public service space remodeling based on service design and behavioral maps. Journal of Industrial and Production Engineering, Abingdon, v. 31, n. 2, p. 76-84, 2014.

WELLINGS, T.; WILLIAMS, M.; TENNANT, C. Understanding customers' holistic perception of switches in automotive human-machine interfaces. Applied Ergonomics, Amsterdam, v. 41, n. 1, p. 8-17, 2010

XU, W. Enhanced ergonomics approaches for product design: A user experience ecosystem perspective and case studies. Ergonomics, Abingdon, v. 57, n. 1, p. 34-51, 2014.
Marcelo Pereira Demilis marcelodemilis@gmail.com

Richard Perassi Luiz de Sousa richard.perassi@uol.com.br

Giselle Alves Diaz Merino gisellemerino@gmail.com 